\title{
Epidemiologische Entwicklung der außerklinischen Beatmung: Eine rasant zunehmende Herausforderung für die ambulante und stationäre Patientenversorgung
}

\section{Evolving Epidemiology of Home Mechanical Ventilation: A Rapidly Growing Challenge for Patient Care}

\section{(c) (1) $\$$}

Authors

C. Karagiannidis ${ }^{1}$, S. Strassmann ${ }^{1}$, J. Callegari ${ }^{1}$, M. Kochanek ${ }^{2}$, U. Janssens ${ }^{3}$, W. Windisch ${ }^{1}$

Institute

1 Lungenklinik Köln-Merheim, ARDS- und ECMO-Zentrum, Kliniken der Stadt Köln gGmbH, Köln, Universität Witten/Herdecke, Fakultät für Gesundheit/Department für Humanmedizin

2 Klinik I für Innere Medizin (Hämatologie und Onkologie), Universitätsklinikum Köln, Köln

3 Klinik für Innere Medizin und Internistische Intensivmedizin St.-Antonius-Hospital Akademisches Lehrkrankenhaus der RWTH Aachen

\section{Bibliografie}

DOI https://doi.org/10.1055/a-0976-9119 |

Pneumologie 2019; 73: 670-676

(c) Georg Thieme Verlag KG Stuttgart · New York

ISSN 0934-8387

Zitierweise für diesen Artikel Dtsch Med Wochenschr 2019; 144: e58-e63, DOI: 10.1055/a-0758-4512

Korrespondenzadresse

Prof. Dr. med. Christian Karagiannidis, ARDS- und ECMO-

Zentrum Köln-Merheim, Lungenklinik Merheim; Kliniken

der Stadt Köln gGmbH, Ostmerheimer Straße 200,

51109 Köln

Christian.Karagiannidis@uni-wh.de

\section{ZUSAMMENFASSUNG}

Hintergrund Die außerklinische Beatmung erfährt in Deutschland eine rasante Entwicklung. Patienten mit chronischer respiratorischer Insuffizienz werden in deutlich zunehmender Zahl nichtinvasiv oder invasiv in einem häuslichen Setting beatmet. Diese Patienten müssen im Rahmen von Kontrolluntersuchungen oder Akut-Problemen immer wieder stationär behandelt werden. Die für die Steuerung des Gesundheitswesens wichtige Entwicklung der stationären Behandlungsfälle ist bislang ebenso unbekannt wie die Morbidität und Krankenhaussterblichkeit der häuslich beatmeten Patienten.

Methode Von 2006-2016 wurden alle stationären Aufnahmen analysiert, bei denen eine Langzeit-Abhängigkeit vom Respirator (mindestens 3 Monate) bestand (Daten des Deutschen Statistischen Bundesamtes).

Ergebnisse Es zeigte sich eine exponentielle Zunahme von stationären Behandlungsfällen, wobei 2006 noch 24845 Patienten stationär behandelt werden mussten, im Jahr 2016 hingegen 86117 Patienten. Im gleichen Zeitraum zeigte sich eine Abnahme der Krankenhaussterblichkeit von 13,2\% (2006) auf 5,7\% (2016). Im Jahr 2016 wurden zudem 47\% der Patienten auf einer Intensiv-, Weaning- oder Überwachungsstation behandelt. Es bestand eine Vielzahl von internistischen und neurologischen Komorbiditäten, welche in Verbindung mit der chronischen respiratorischen Insuffizienz die große Patientenkohorte als schwer krankes Patientenkollektiv charakterisierte. Führende Diagnosen waren die COPD mit $58 \%$ und eine Vielzahl kardiologischer Diagnosen. Auffällig viele Patienten (24\%) zeigten eine Einschränkung der Nierenfunktion bis hin zur Dialysepflichtigkeit.

Diskussion Die rasante Zunahme der außerklinischen Beatmung hat wesentlichen Einfluss auf die Entwicklung der Krankenhauslandschaft in Deutschland. Der exponentielle Anstieg der sehr pflegeintensiven Patienten stellt das Gesundheitssystem vor extreme Herausforderungen und erfordert eine gesundheitspolitische Diskussion über die Grenzen des Systems.

\section{ABSTRACT}

Background Home mechanical ventilation is dramatically evolving in Germany. Patients with non-invasive and invasive ventilation are increasingly treated at home. In-hospital treatment of these patients is also necessary either for control visits or the management of acute medical problems. However, the development of in-hospital patient care, morbidity and mortality of these patients is unknown.

Methods All patients with long-term dependence on mechanical ventilation for more than three months requiring hospitalisation between 2006 and 2016 were analysed (data obtained from the Federal Statistical Office of Germany).

Results There was an exponential increase in the number of in-patients with long-term dependence of mechanical venti- 
lation. While 24845 patients were treated in-hospital in 2006, 86117 patients were treated in 2016. Correspondingly, mortality decreased from $13.2 \%$ (2006) to $5.7 \%$ (2016). In addition, in $201647 \%$ of all patients were treated on the intensive care or high dependency care unit. Overall, patients had been severely ill, as there were plenty of medical and neurological co-morbidities. The most common diagnosis was COPD with $58 \%$ of all cases, followed by several cardi- ology diagnosis. A high number of patients had an impairment of renal function (24\%), in part requiring dialysis.

Conclusions The rapid development of home mechanical ventilation substantially impacts on the development of the hospital landscape in Germany. The exponential increase of these care-intensive patients is challenging for the health care system and requires a discussion about its limits.

\section{Einleitung}

Die maschinelle Beatmung gehört heute zu den etablierten Standardverfahren in der Behandlung der respiratorischen Insuffizienz. Eine invasive oder nichtinvasive Beatmung (NIV) kommt sowohl bei akuter respiratorischer Insuffizienz im Rahmen einer Notfallbehandlung als auch lebenserhaltend in der Langzeitbeatmung im außerklinischen Setting zum Einsatz. Eine außerklinische Langzeitbeatmung kann als elektive Maßnahme bei nicht mehr beherrschbarer chronischer respiratorischer Insuffizienz mit Hyperkapnie als Ausdruck eines chronischen Versagens der Atempumpe eingeleitet werden [1]. Grundsätzlich wird versucht, der NIV bei chronisch respiratorischer Insuffizienz den Vorzug zu geben, während eine elektive Tracheotomie zur Langzeitbeatmung nur selten erfolgt. Idealerweise erfolgt die Tracheotomie bei Patienten mit neuromuskulären Erkrankungen, deren Schluckakt und/oder Sekretelimination zusätzlich zur respiratorischen Insuffizienz gestört sind [1]. Häufiger jedoch resultiert eine invasive Langzeitbeatmung aus einem lang andauernden intensivmedizinischen Aufenthalt. Viele dieser Patienten haben eine zugrunde liegende chronische respiratorische Störung oder wesentliche Komorbiditäten und/oder einen schweren Krankheitsverlauf mit langen Beatmungszeiten auf der Intensivstation mit entsprechenden ungünstigen Langzeitfolgen der Muskel- und Atmungsfunktion [2]. Die außerklinische invasive Beatmung ist zudem unter ökonomischen Gesichtspunkten mit sehr hohen Therapiekosten von etwa 250000 Euro/Jahr/Patient und Gesamtkosten für das Gesundheitssystem von mehr als 4 Mrd. Euro/Jahr verbunden [3].

Vor der Verlegung in eine außerklinische Beatmung sollte bei Schwierigkeiten in der Entwöhnung vom Respirator nach aktuellen Empfehlungen möglichst eine Verlegung in ein zertifiziertes Weaning-Zentrum erfolgen [2]. Dabei handelt es sich um Fachabteilungen mit spezieller Ausrichtung auf Patienten mit prolongiertem Weaning, also solche, die trotz grundsätzlicher Möglichkeit zur Entwöhnung auch länger als 1 Woche nach Etablierung einer Entwöhnungs-Bereitschaft dennoch nicht entwöhnbar sind [2, 4, 5]. In diesen Zentren gelingt es immerhin bei ca. 43 \% der Patienten, eine Entlassung ohne weiterführende Beatmung zu gewährleisten. Bei 19\% gelingt dies aber nur, indem die Patienten häuslich eine NIV weiterführen, während ca. $23 \%$ der Patienten eine invasive Langzeitbeatmung benötigen [5].

Basierend auf aktuellen wissenschaftlichen Arbeiten und in Anlehnung an die Bedingungen und Voraussetzungen im deut- schen Gesundheitssystem bezüglich der Versorgungsstrukturen hat die Deutsche Gesellschaft für Pneumologie und Beatmungsmedizin e. V. (DGP) im Verbund mit anderen Fachgesellschaften Leitlinien für die invasive und nichtinvasive außerklinische Beatmung [1] sowie für das prolongierte Weaning [2] publiziert.

Besonders in den letzten Jahren ist die Anzahl der Patienten mit außerklinischer Beatmung stark ansteigend. Exakte Zahlen oder Erhebungen existieren in Deutschland allerdings nicht. Unklar ist auch die Verteilung der Patienten mit invasiver oder nichtinvasiver Beatmung. Zudem ist der Einfluss der Zunahme von Patienten mit außerklinischer Beatmung auf die weiterführende stationäre Behandlung nicht geklärt. Hier empfiehlt die deutsche Leitlinie, dass außerklinisch beatmete Patienten regelmäßig zu stationären Kontrolluntersuchungen vorstellig werden müssen [1]. Zudem erfolgen stationäre Aufnahmen außerhalb der geplanten Kontrolluntersuchungen bei Schwierigkeiten mit der außerklinischen Beatmung und anderen akuten, stationär zu behandelnden Erkrankungen.

Dabei ist es unklar, ob Patienten mit etablierter außerklinischer Beatmung im Falle einer stationären Versorgung auf einer Intensivstation/Überwachungsstation behandelt werden können, oder ob hier Behandlungen auch auf Normalstationen möglich wären. Studien zu den Intensivkapazitäten in Deutschland haben in diesem Zusammenhang gezeigt, dass es zwar einen Rückgang der Gesamtkrankenhausbetten in Deutschland gibt, allerdings gleichzeitig die Intensivbettenkapazität steigend ist [6]. Dabei verfügt Deutschland auch im internationalen Vergleich über die meisten Intensivbetten pro Einwohner [7]. In Anbetracht eines stark aggravierten Intensivpflegemangels und der damit verbundenen Bettensperrungen stellen die rasant wachsenden Zahlen stationärer Aufnahmen dieser Patienten eine besondere Herausforderung dar [8].

Die mutmaßlich stark steigende Zahl außerklinisch beatmeter Patienten ist somit auch in Bezug auf intermittierend notwendige stationäre Krankenhausbehandlungen von fundamentalem Interesse, insbesondere auch vor dem Hintergrund des Kostendrucks im Gesundheitssystem und auch der weiteren Planung einer Krankenhauslandschaft. Das Ziel der vorliegenden Studie war daher, die epidemiologische Entwicklung der stationären Behandlungen von Patienten mit Langzeitbeatmung im Verlauf der letzten 10 Jahre zu skizzieren und im Weiteren, insbesondere im Hinblick auf notwendige Prozeduren und Komorbiditäten, zu charakterisieren. 


\section{Methodik}

Alle Daten wurden vom Deutschen Statistischen Bundesamt in Wiesbaden zur Verfügung gestellt. Nach § 21 des Krankenhausentgeltgesetzes sind alle voll- und teilstationären Einrichtungen in Deutschland verpflichtet, umfangreiche Leistungsdaten u. a. mit Diagnosen und Prozeduren an das statistische Bundesamt zu übermitteln. Diese Daten können in anonymisierter Form beim statistischen Bundesamt nach dem entsprechenden ICD- und/oder OPS-Code abgefragt werden. Ein Rückschluss auf einzelne Patienten oder Einrichtungen ist explizit nicht möglich. Für diese Analyse wurde als primäre Grundlage der Operationen- und Prozeduren-Schlüssel (OPS) Z99 zugrunde gelegt: Abhängigkeit (langzeitig) von unterstützenden Apparaten, medizinischen Geräten oder Hilfsmitteln, anderenorts nicht klassifiziert. Hier wurde primär die Z99.1 (Abhängigkeit vom Respirator) untersucht und die Kombination dieser OPS mit allen ICD-Codes der Jahre 2005-2016 abgefragt. Eine Langzeit-Abhängigkeit vom Respirator (invasiv und nichtinvasiv) ist im DRG-System definiert als eine Vollendung einer nichtinvasiven oder invasiven Beatmungstherapie von mindestens 3 Monaten Dauer. Eine Unterscheidung der stationären Aufnahmen zwischen Akutaufnahmen und Kontrolluntersuchungen beziehungsweise mit oder ohne invasive Beatmung wird aufgrund der ICD/OPS erst in Zukunft möglich sein und lässt sich an Hand der retrospektiven Daten nur eingeschränkt und unvollständig erheben.

Es wurde entsprechend der letzten 10 verfügbaren Jahre ein Analysezeitraum von 2006-2016 gewählt. Im Weiteren wurden wesentliche Haupt- und Nebendiagnosen miterfasst, wobei hier im Wesentlichen zwischen neurologischen und internistischen/allgemeinmedizinischen Diagnosen unterschieden worden war. Explizit erfolgte die Analyse der Haupt- und Nebendiagnosen für das aktuelle Jahr 2016, gleichwohl die Ergebnisse repräsentativ auch für die Vorjahre gewesen waren. Eine Unterscheidung zwischen Haupt- und Nebendiagnose wurde nicht getroffen, da es bei der Analyse unklar bleiben musste, welche Diagnose medizinisch respektive aus abrechnungsrelevanten Gründen im Vordergrund stand. Während erlösrelevante Prozeduren im Gesundheitswesen in der Regel vollständig kodiert werden, ist dies bei nicht erlösrelevanten Diagnosen nicht immer zwingend der Fall.

\section{Ergebnisse}

Als wesentliches Ergebnis konnte gezeigt werden, dass die Zahl der Langzeitbeatmungspatienten, welche in einer Klinik aufgenommen werden mussten, über den Zeitraum von 2006 bis 2016 stark und exponentiell ansteigend war. So wurden alleine im Jahr 2016 insgesamt 86117 Patienten mit etablierter außerklinischer Beatmung erneut stationär behandelt ( $>$ Abb. 1). Im Jahr 2006 waren dies nur 24845 Patienten. Bemerkenswert erscheint zudem, dass knapp 1500 Patienten zwischen 90 und 100 Jahre alt waren. Die Mortalität der Patienten reduzierte sich deutlich von maximal 13,2\% im Jahr 2006 auf aktuell minimal 5,7\% im Jahr 2016 (ฉ Abb. 2).

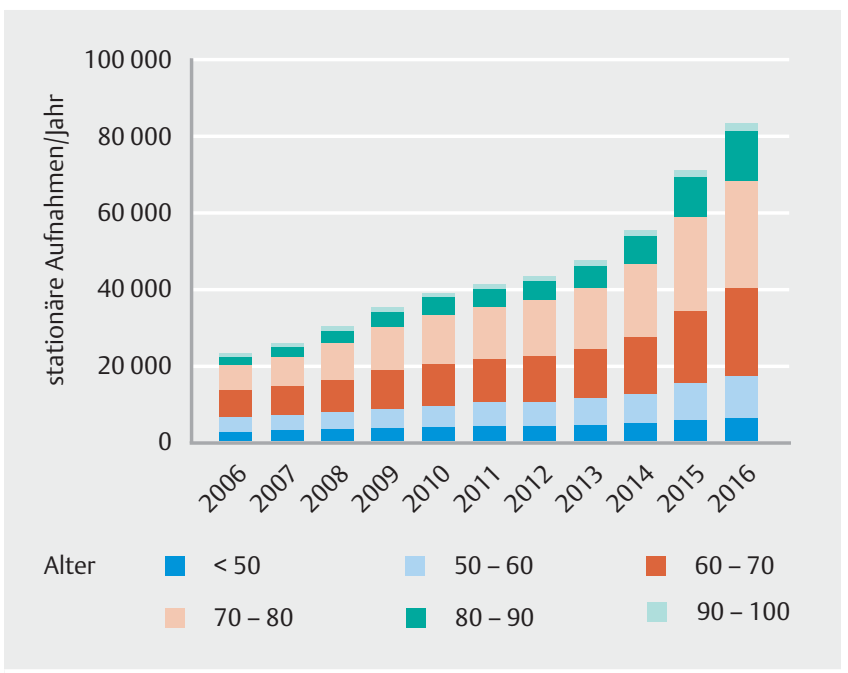

- Abb.1 Anzahl der stationären Aufnahmen mit Verschlüsselung der Z99.1 (Abhängigkeit vom Respirator) im Verlauf von 2006 bis 2016 nach Alter.

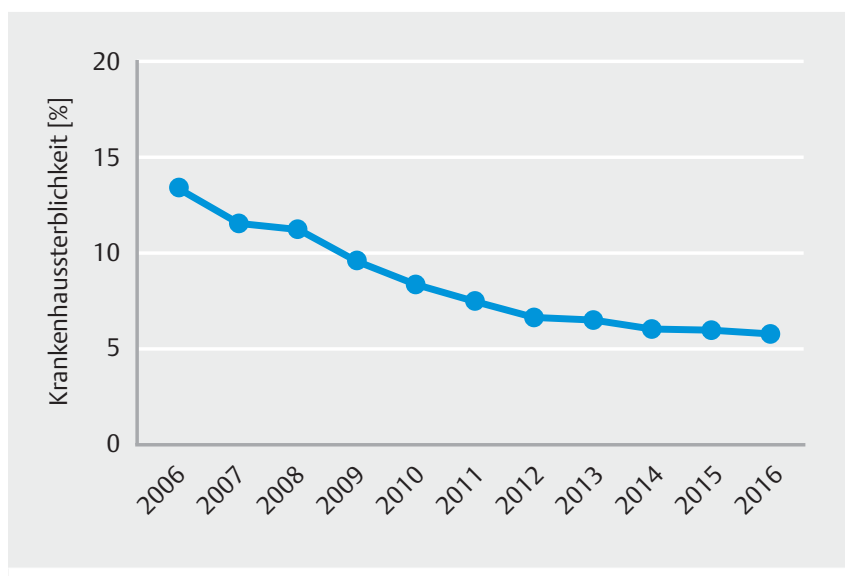

- Abb.2 Mortalität der Patienten mit Verschlüsselung der Z99.1 (Abhängigkeit vom Respirator) im Verlauf von 2006 bis 2016.

Im Jahr 2016 sind 23\% aller stationär aufgenommenen Patienten auf einer Intensivstation behandelt worden, während $24 \%$ der Patienten zumindest auf einer Monitoring-Station, wie z. B. einer Weaning- oder IMC-Station, versorgt worden sind. Folglich wurden 53 \% der Patienten auf einer Normalstation betreut. Weitere Angaben zur Häufigkeit resistenter Erreger und Therapieverfahren (Tracheotomie, Dialyse, extrakorporale Membran-Oxygenierung) sind in $>$ Tab. 1 dargestellt.

Wie aus > Tab. 2 ersichtlich ist, bestanden eine Reihe von pneumologischen und kardiologischen Diagnosen. So hatten $58 \%$ der Patienten eine COPD, 32\% eine Herzinsuffizienz und $25 \%$ eine chronische ischämische Herzerkrankung, während $32 \%$ einen Diabetes mellitus Typ II aufwiesen und 24\% der Patienten eine chronische Niereninsuffizienz. Bei $23 \%$ der Patienten wurde eine Adipositas kodiert. In $12 \%$ der Fälle lag bei Aufnahme eine Pneumonie vor, bei 8,7\% ein isolationspflichtiger Krankheitserreger. 
- Tab. 1 Relevante intensivmedizinische Prozeduren im Jahr 2016, Mehrfachnennungen möglich $(n=86117)$.

\begin{tabular}{|l|c|c|}
\hline Behandlung auf einer Intensivstation & 19717 & $(23 \%)$ \\
\hline $\begin{array}{l}\text { Behandlung auf einer Monitoring-Station, } \\
\text { z. B.: RICU, Weaning-Station, IMC }\end{array}$ & 20695 & $(24 \%)$ \\
\hline $\begin{array}{l}\text { Erreger mit bestimmten Antibiotika-Resis- } \\
\text { tenzen, die besondere therapeutische oder } \\
\text { hygienische Maßnahmen erfordern }\end{array}$ & 7525 & $(8,7 \%)$ \\
\hline $\begin{array}{l}\text { Tracheotomie } \\
\text { Nierenersatzverfahren }\end{array}$ & 4228 & $(5 \%)$ \\
\hline ECMO-Therapie & 2265 & $(2,6 \%)$ \\
\hline
\end{tabular}

Wesentlich ist aber auch das Vorliegen relevanter neurologischer Diagnosen ( $\vee$ Tab.3). So fanden sich bei knapp 10\% der Patienten motorische Funktionseinschränkungen und bei 8,3\% der Patienten eine Depression. Zwar war der prozentuale Anteil von Patienten mit anoxischer Hirnschädigung $(2,2 \%)$ augenscheinlich nicht im hohen Prozentbereich. Die Absolut-Zahl von Patienten $(n=1886)$ zeigt aber beispielhaft die Dimensionen der zugrunde liegenden neurologischen Erkrankungen.

\section{Diskussion}

Die vorliegende Auswertung zu stationär aufgenommen Patienten mit invasiver oder nichtinvasiver Langzeitbeatmung kommt zu folgenden Kernaussagen:

1. Die Zahl von Patienten mit außerklinischer Beatmung, die im Verlauf eine stationäre Behandlung benötigen, ist über die letzten 10 Jahre rasant und exponentiell ansteigend.

2. Dabei nimmt insbesondere die Zahl älterer Patienten mit einem Alter von über 60 Jahren deutlich zu. Hierbei nimmt die Krankenhaussterblichkeit sukzessive über die Jahre ab und liegt 2016 nur noch bei 5,7\%.

3. Es handelt sich bei diesen Patienten um komplex kranke Menschen mit einer hohen Komorbidität und Einschränkungen einer Organfunktion auf verschiedensten Ebenen, die sowohl im ambulanten als auch stationären Setting verschiedene Fachdisziplinen mit der entsprechenden Expertise erfordern.

4. Neben einer respiratorischen Insuffizienz, einer kardialen Komorbidität, infektiologischen Erkrankungen mit teils multiresistenten Erregern und einer Niereninsuffizienz sind insbesondere schwere neurologische Einschränkungen koexistent.

Die vorliegenden Ergebnisse haben weitreichende Implikationen für die Entwicklung der intensiv- und notfallmedizinischen Krankenversorgung in Deutschland und werfen zudem gravierende weiterführende Fragen auf. Basierend auf dem Trend der aktuellen Daten muss für die Zukunft mit einer weiteren dramatischen Zunahme dieser Patienten auch im stationären Sektor gerechnet werden. Dies stellt die deutsche Krankenhauslandschaft und auch die ökonomischen Ressourcen vor eine schwerwiegende Herausforderung. In diesem Sinne bleibt nicht
- Tab. 2 Relevante internistische Haupt- oder Nebendiagnosen im Jahr 2016, Mehrfachnennungen möglich ( $n=86117)$.

\begin{tabular}{|c|c|c|}
\hline \multicolumn{3}{|l|}{ pneumologische Diagnosen } \\
\hline respiratorische Insuffizienz & 57623 & $(67 \%)$ \\
\hline COPD & 49986 & $(58 \%)$ \\
\hline Pneumonie & 10228 & $(12 \%)$ \\
\hline Pleuraerguss & 8231 & $(10 \%)$ \\
\hline Nikotinabusus & 4017 & $(4,7 \%)$ \\
\hline Lungenkarzinom & 3051 & $(3,5 \%)$ \\
\hline Asthma bronchiale & 1519 & $(1,8 \%)$ \\
\hline Blutung aus den Atemwegen & 1306 & $(1,5 \%)$ \\
\hline Lungenembolien & 769 & $(<1 \%)$ \\
\hline Pneumothorax & 560 & $(<1 \%)$ \\
\hline \multicolumn{3}{|l|}{ kardiologische Diagnosen } \\
\hline essentielle Hypertonie & 44399 & $(52 \%)$ \\
\hline $\begin{array}{l}\text { Dauertherapie (gegenwärtig) } \\
\text { mit Antikoagulanzien }\end{array}$ & 28318 & $(33 \%)$ \\
\hline Herzinsuffizienz & 27592 & $(32 \%)$ \\
\hline Vorhofflimmern & 21845 & $(25 \%)$ \\
\hline chronische ischämische Herzkrankheit & 21444 & $(25 \%)$ \\
\hline hypertensive Herzkrankheit & 9970 & $(12 \%)$ \\
\hline Kardiomyopathie & 2191 & $(2,5 \%)$ \\
\hline akuter Myokardinfarkt & 1572 & $(1,8 \%)$ \\
\hline $\begin{array}{l}\text { Herzstillstand mit erfolgreicher und } \\
\text { nicht erfolgreicher Reanimation }\end{array}$ & 1450 & $(1,8 \%)$ \\
\hline \multicolumn{3}{|l|}{ allgemein-internistische Diagnosen } \\
\hline Diabetes Mellitus Typ ॥ & 27549 & $(32 \%)$ \\
\hline $\begin{array}{l}\text { chronische Niereninsuffizienz Stadium I-V } \\
\text { und chronisches Nierenversagen }\end{array}$ & 21020 & $(24 \%)$ \\
\hline Adipositas & 19887 & $(23 \%)$ \\
\hline Probleme in Bezug auf Pflegebedürftigkeit & 15461 & $(18 \%)$ \\
\hline Versorgung künstlicher Körperöffnungen & 9617 & $(11 \%)$ \\
\hline akutes Nierenversagen & 6074 & $(7,1 \%)$ \\
\hline Stuhlinkontinenz & 5706 & $(6,6 \%)$ \\
\hline bösartige Neubildung in der Eigenanamnese & 5153 & $(6,0 \%)$ \\
\hline Dekubital-Geschwür und Druckzone & 4654 & $(5,4 \%)$ \\
\hline $\begin{array}{l}\text { Vorhandensein einer künstlichen } \\
\text { Körperöffnung }\end{array}$ & 4064 & $(4,7 \%)$ \\
\hline
\end{tabular}

nur die Verfügbarkeit von Betten auf einer Intensiv- oder Überwachungsstation zu diskutieren [6], sondern auch die Versorgungsqualität. Wie dargestellt, verlangt die Vielzahl von zugrunde liegenden Haupt- und Nebendiagnosen die Expertise unterschiedlichster Fachdisziplinen - und dies in der ambulanten und stationären Versorgung. Der zunehmenden Zahl 
- Tab. 3 Relevante Neurologische Haupt- oder Nebendiagnosen im Jahr 2016, Mehrfachnennungen möglich $(n=86117)$.

\begin{tabular}{|l|c|c|}
\hline motorische Funktionseinschränkungen & 8166 & $(9,5 \%)$ \\
\hline Depression & 7175 & $(8,3 \%)$ \\
\hline Epilepsie & 3011 & $(3,5 \%)$ \\
\hline $\begin{array}{l}\text { Paraparese und Paraplegie, Tetraparese } \\
\text { und Tetraplegie }\end{array}$ & 3004 & $(3,5 \%)$ \\
\hline $\begin{array}{l}\text { extrapyramidale Bewegungsstörungen } \\
\text { Demenz }\end{array}$ & 2895 & $(3,4 \%)$ \\
\hline Polyneuropathien & 2775 & $(3,2 \%)$ \\
\hline Hemiparese und Hemiplegie & 2510 & $(2,9 \%)$ \\
\hline kognitive Funktionseinschränkung & 2493 & $(2,9 \%)$ \\
\hline $\begin{array}{l}\text { primäre Myopathien, z. B. Duchenne-Muskel- } \\
\text { dystrophie }\end{array}$ & 1960 & $(2,3 \%)$ \\
\hline \begin{tabular}{l} 
anoxische Hirnschädigung, Hirnödem \\
\hline Skoliose
\end{tabular} & 1886 & $(2,2 \%)$ \\
\hline $\begin{array}{l}\text { spinale Muskelatrophie und verwandte } \\
\text { Syndrome }\end{array}$ & 1771 & $(2,0 \%)$ \\
\hline Hirninfarkt & 1642 & $(1,9 \%)$ \\
\hline \begin{tabular}{l} 
Status epilepticus \\
\hline
\end{tabular} & 1061 & $(1,2 \%)$ \\
\hline & 236 & $(<1 \%)$ \\
\hline
\end{tabular}

höchstaufwendiger Patienten mit der häufigen Notwendigkeit einer 24-stündigen außerklinischen Intensivbetreuung steht der zunehmend dramatisch werdende Pflegepersonalmangel gegenüber [8]. Je größer diese Schere wird, desto mehr ist zu befürchten, dass die Versorgungsqualität und die grundsätzliche Möglichkeit der Versorgung dieser Patienten darunter leiden. Dabei ist bereits heute bekannt, dass die Zahl der Intensivpflegedienste alleine zwischen Januar 2014 und Dezember 2016 um fast $25 \%$ zugenommen hat, was im Vergleich zu allen ambulanten Pflegediensten damit überproportional ist [9]. Wie auch für die stationäre Versorgung sollten zur Aufrechterhaltung der Qualität ebenfalls im ambulanten Bereich Personaluntergrenzen und eine permanente Betreuung durch Fachpflege 24/7 gefordert werden. Der ambulante Bereich entzieht sich jedoch leider häufig einer dringend notwendigen Kontrolle der Versorgungsstrukturen.

Wesentlich erscheint auch die Beobachtung, dass insbesondere eine deutliche Zunahme von älteren Patienten mit Langzeitbeatmung besteht. So konnte bereits vor einigen Jahren gezeigt werden, dass in spezialisierten Weaning-Zentren trotz steigender Expertise und Erfahrung das Weaning-Outcome über die Jahre abgenommen hat, was durch das zunehmende Alter und die Zunahme der Komorbiditäten bei diesen Patienten erklärt werden konnte [10]. Eine Beatmungstherapie bei Menschen im fortgeschrittenen Alter mit vielen Komorbiditäten wirft allerdings auch erhebliche ethische Fragen auf. So konnten aktuelle wissenschaftliche Studien zeigen, dass im Falle eines erfolglosen Weanings die Fortsetzung einer invasiven Langzeitbeatmung im außerklinischen Setting mit einer äußerst reduzierten Lebensqualität verbunden sein kann [11] [12]. In diesem Sinne ergibt sich die Frage, ob alles das, was die moderne Medizin heute leisten kann, unter ethischen Betrachtungen auch geleistet werden muss. Es sei an dieser Stelle angemerkt, dass sich nach komplexen intensivmedizinischen Behandlungen oftmals lange Behandlungszeiten auf einer Entwöhnungseinheit anschließen, was für die Patienten eine hohe physische und psychische Belastung darstellt. Der reine Fokus auf die medizinisch komplexen Zusammenhänge lässt aber nicht selten die wesentlichen Therapieziele aus Sicht der Patienten außer Acht. Bezüglich detaillierter Ausführungen zur Therapiezielfindung, Therapiezieländerung und der entsprechenden Kommunikation innerhalb und außerhalb des Behandlungsteams sei an dieser Stelle auf das Positionspapier „Therapiezieländerung und Therapiebegrenzung in der Intensivmedizin “ der Sektion Ethik der Deutschen Interdisziplinären Vereinigung für Intensiv- und Notfallmedizin (DIVI) verwiesen [13].

Im Falle einer Nicht-Entwöhnbarkeit erfolgt die Überleitung in die außerklinische Beatmung $[1,2]$. Hier kommt in der Regel entweder eine Betreuung im eigenen häuslichen Umfeld mit einer häuslichen Intensivpflege in Betracht oder alternativ eine Unterbringung in einem Beatmungspflegeheim oder in einer Beatmungs-Wohngemeinschaft. Die Prozess-Abläufe von der Notaufnahme über die Intensivstationen, die Weaning-Stationen und die Normalstationen in die Häuslichkeit sind vielschichtig und komplex. Sie integrieren in der Regel eine Vielzahl von unterschiedlichen Fachabteilungen und auch Ärzten. Dabei reflektiert die Tatsache, dass ein wesentlicher Anteil der Patienten über 80 und gar 90 Jahre alt ist, den Umstand, dass die Medizin an dieser Stelle grenzenlos ist. Hier muss zudem auch herausgestellt werden, dass die ambulante ärztliche Betreuung im außerklinischen Setting bis dato nicht zufriedenstellend geregelt ist, da es hierfür keine fachärztliche Betreuung bzw. entsprechende Versorgungsstruktur gibt $[1,11,12]$. Hier wären ein Modell spezieller ambulanter Versorgung, analog zur speziell ambulanten palliativmedizinischen (SAPV) Versorgung, oder telemedizinische Projekte denkbar. In jedem Fall sollte eine optimierte ambulante Struktur die Zahl der stationären Einweisungen zu minimieren versuchen.

Natürlich muss auch die gängige Praxis der Beatmung und der teils unkritischen Transfers in die außerklinische Beatmung kritisch hinterfragt werden. Ist es ethisch richtig, Patienten mit schwerem hypoxischem Hirnschaden oder gar appalische Patienten in eine außerklinische Beatmung zu entlassen? Handelt es sich hier eventuell auch bei fehlendem Therapieziel um eine Übertherapie, die im schlimmsten Fall noch ökonomischen Interessen unterliegt? Dies insbesondere auch im Hinblick auf ein Urteil des Oberlandesgerichts München (Dezember 2017), das eine Übertherapie gar als Behandlungsfehler eingestuft hat. Diese drängenden Fragen müssen zeitnah unter Einbeziehung des Gesetzgebers im Rahmen einer gesellschaftlichen ethischen Diskussion gelöst werden.

Es bleibt im Rahmen der vorliegenden Studie im Detail unklar, was primäre Aufnahmegründe für die stationäre Behandlung sind, da dies nicht immer über die Hauptdiagnose bei Entlassung abgebildet ist. Der hohe Anteil an Patienten in der aktuellen Analyse, die auf einer Intensivstation oder Überwachungs- 
station (zusammen 47\%) behandelt worden sind, legt nahe, dass akute, im außerklinischen Setting nicht lösbare Probleme aufgetreten waren. Vorstellbar ist allerdings auch, dass mangelnde Verfügbarkeit und Expertise im außerklinischen Setting zu stationären Einweisungen führten. Dies bleibt für die Zukunft weiter zu untersuchen.

Schließlich besteht auch die Frage, ob Kontrolluntersuchungen einer außerklinischen Beatmung zwingend stationär durchgeführt werden müssen. Hier positioniert sich die DGP insofern eindeutig, als dass es für ambulante Kontrolluntersuchungen bis dato nur wenig Evidenz gibt, während auch hier entsprechende Versorgungsstrukturen in keinster Weise flächendeckend vorgehalten werden können, sodass die primäre stationäre Kontrolluntersuchung einer außerklinischen Beatmungstherapie weiterhin in den Leitlinien empfohlen wird [1]. Es findet sich allerdings eine große Bandbreite von Beatmungspatienten. Komplexe Patienten mit Tracheostoma und ausgewiesener Komorbidität bedürfen einer anderen Kontrolle ihrer Beatmungstherapie als Patienten ohne wesentliche Komorbiditäten, die eine Beatmungsmaske (NIV) selbstständig an- und ablegen können. Hier zeigen erste Daten auch aus Deutschland, dass wahrscheinlich nicht alle Patienten mit Langzeit-NIV wirklich einer stationären Kontrolle bedürfen [14]. Zudem erwähnt die Leitlinie, dass es hier in der Zukunft grundsätzlich wünschenswert wäre, dass die Sektoren der ambulanten und stationären Patientenversorgung an der Schnittstelle der Beatmungsmedizin enger aneinanderrücken, als dies aktuelle Strukturen im Gesundheitssystem vorsehen [1]. Insofern bietet die aktuelle Arbeit Informationen, die nicht nur für Mediziner unterschiedlicher Fachrichtungen wegweisend sind, sondern auch für die Vertreter der Gesundheitsökonomie und der Gesundheitspolitik zur weiteren Diskussion dieser Fragen wichtig erscheinen. Insbesondere muss angemerkt werden, dass die außerklinische Beatmung kaum einer Regulierung unterliegt und im Gegensatz zur stationären Versorgung nicht vom medizinischen Dienst der Krankenkassen kontrolliert wird.

Abschließend sei angemerkt, dass eine Betreuung von Beatmungspatienten sowohl in der Klinik als auch im außerklinischen Setting ökonomischen Anreizen unterlegen ist. Selbstverständlich sind die Autoren der aktuellen Arbeit der Meinung, dass eine Beatmungsmedizin auf höchster Qualitätsstufe durchgeführt und dies entsprechend entlohnt werden muss. Eine dramatische Zunahme von Beatmungsfällen in und außerhalb der Klinik darf jedoch nicht zu einem Qualitätsverlust in der Behandlung dieser Patienten führen, wenn ökonomische Limitationen konsekutiv Einbußen in der Behandlungsqualität nach sich ziehen. Vor diesen Hintergründen ist es an der Zeit, dass wir die Entwicklungen in der Beatmungsmedizin in Deutschland mit rasant steigenden Patientenzahlen kritisch bewerten und dass wir in Anbetracht medizinischer, ethischer und ökonomischer Aspekte bewusste Entscheidungen für diesen speziellen und wichtigen Bereich in der Medizin treffen. Hier ist die großflächige prospektive Versorgungsforschung dringend gefordert, verlässliche Daten zu generieren, insbesondere im Hinblick auf die Lebensqualität dieser Patientengruppe, und auch die kritische Frage zu stellen, ob insbesondere tracheotomierte Langzeit-Beatmungs-Patienten den Weg der Tracheoto- mie nochmals wählen würden, wenn sie selbst hätten entscheiden können. Wichtig wäre daher vor Verlegung in eine außerklinische Beatmung für jeden Patienten eine gesetzlich verpflichtende ethische Fallbesprechung in einem strukturierten Dialog aller beteiligten Akteure nebst externem Sachverständigen.

\section{KERNAUSSAGEN}

- Zwischen 2006 und 2016 zeigt sich eine exponentielle Zunahme von stationären Behandlungsfällen langfristig außerklinisch beatmeter Patienten (von n= 24845 auf $\mathrm{n}=86117)$.

- Im gleichen Zeitraum zeigte sich eine Abnahme der Krankenhaussterblichkeit von 13,2\% (2006) auf 5,7\% (2016).

- Im Jahr 2016 wurden 47\% der Patienten auf einer Intensiv- oder Überwachungsstation behandelt.

- Es bestand eine Vielzahl von internistischen und neurologischen Komorbiditäten, welche die große Patientenkohorte als schwer krankes Patientenkollektiv charakterisierten.

- Die führenden Diagnosen und Komorbiditäten waren die COPD bei $58 \%$ aller Patienten, eine Herzinsuffizienz bei $32 \%$, eine chronische ischämische Herzerkrankung bei $25 \%$, ein Diabetes mellitus Typ II bei $32 \%$ und eine chronische Niereninsuffizienz in $24 \%$ der Fälle.

Interessenkonflikt

Die Autoren geben an, dass kein Interessenkonflikt besteht.

Literatur

[1] Windisch W, Dreher M, Geiseler J et al. Guidelines for Non-Invasive and Invasive Home Mechanical Ventilation for Treatment of Chronic Respiratory Failure - Update 2017. Pneumologie 2017; 71: 722 - 795

[2] Schonhofer B, Geiseler J, Dellweg D et al. Prolonged weaning: S2k-guideline published by the German Respiratory Society. Pneumologie 2014; 68: 19-75

[3] (DIGAB) DIGfAB. Stellungnahme der Deutschen Interdisziplinären Gesellschaft für Außerklinische Beatmung (DIGAB e.V.) zu den Ausführungen von Dr. Matthias Thöns aus Witten auf dem Bremer Palliativkongresses am 20. März 2015. In; 2015

[4] Boles JM, Bion J, Connors A et al. Weaning from mechanical ventilation. Eur Respir J 2007; 29: 1033-1056

[5] WeanNet Study G. WeanNet: The network of weaning units of the DGP (Deutsche Gesellschaft fur Pneumologie und Beatmungsmedizin) - results to epidemiology an outcome in patients with prolonged weaning. Dtsch Med Wochenschr 2016; 141: e166-e172

[6] Thattil R, Klepzig D, Schuster M. Intensive care capacities in Germany: provision and usage between 1991 and 2009. Anaesthesist 2012; 61: $56-62$

[7] Rhodes A, Ferdinande P, Flaatten $\mathrm{H}$ et al. The variability of critical care bed numbers in Europe. Intensive Care Med 2012; 38: 1647-1653 
[8] Karagiannidis C, Kluge S, Riessen R et al. Impact of nursing staff shortage on intensive care medicine capacity in Germany. Med Klin Intensivmed Notfmed 2018. doi:10.1007/s00063-018-0457-3

[9] Meißner S. Marktanalyse Intensivpflege und Intensiv-WG. In 2016

[10] Polverino E, Nava S, Ferrer M et al. Patients' characterization, hospital course and clinical outcomes in five Italian respiratory intensive care units. Intensive Care Med 2010; 36: 137-142

[11] Huttmann SE, Windisch W, Storre JH. Invasive home mechanical ventilation: living conditions and health-related quality of life. Respiration 2015; 89: 312-321
[12] Huttmann SE, Magnet FS, Karagiannidis C et al. Quality of life and life satisfaction are severely impaired in patients with long-term invasive ventilation following ICU treatment and unsuccessful weaning. Ann Intensive Care 2018; 8: 38

[13] Janssens UBH, Duttge G, Erchinger R et al. Therapiezieländerung und Therapiebegrenzung in der Intensivmedizin Positionspapier der Sektion Ethik der DIVI. In 2012

[14] Schwarz SB, Callegari J, Hamm C et al. Is Outpatient Control of LongTerm Non-Invasive Ventilation Feasible in Chronic Obstructive Pulmonary Disease Patients? Respiration 2018; 95: 154-160 